\title{
Correlação entre o número de parafusos e o percentual de correção no tratamento cirúrgico da escoliose neuromuscular
}

Correlation between number of screw and curve correction in the treatment of neuromuscular scoliosis

Correlación entre el número de tornillos y el porcentual de corrección en el tratamiento quirúrgico de la escoliosis neuromuscular

\author{
Murilo Tavares Daher ${ }^{1}$ \\ Paulo Tadeu Maia Cavali2 \\ Marcus Alexandre Mello Santo 2 \\ Alexander Junqueira Rossato ${ }^{2}$ \\ Mauricio Antonelli Lehoczki ${ }^{2}$ \\ Élcio Landim ${ }^{3}$
}

\section{RESUMO}

Objetivo: avaliar se existe relação entre o número de parafusos pediculares (densidade de parafusos) e o percentual de correção da curva principal no tratamento cirúrgico das escolioses neuromusculares. Métodos: foram avaliados, retrospectivamente, 55 pacientes portadores de escoliose neuromuscular submetidos ao tratamento cirúrgico por meio de artrodese exclusivamente pela via posterior. Foram analisados o valor da curva pré-operatória, o percentual de correção e o valor da curva pós-operatória nas radiografias no pré-operatório e no pós-operatório imediato. Foi calculada a densidade de parafuso (número de parafusos por pedículo na área correspondente à curva principal) e avaliada a sua relação com o percentual de correção pela análise de correlação de Spearman. Resultados: dos 55 pacientes, 28 (51\%) eram do sexo feminino e 27 (49\%) do mas-

\begin{abstract}
Objective: to investigate if the number of pedicular screw (screw density) within the major curve correlates with the curve correction in the surgical treatment of neuromuscular scoliosis. Methods: a total of 55 patients with neuromuscular scoliosis that were exclusively submitted to posterior spinal fusion with pedicular screw constructs were retrospectively investigated. Data included major curve measurements assessed on pre and immediate postoperative radiographs, and the percentage of curve correction. The screw density number of screw within the major curve relative to the number of available implant sites) was calculated; its relationship with the percentage of the curve correction was evaluated by the Spearman's rank correlation coefficient. Results: twenty-eight (51\%) females and
\end{abstract}

\section{RESUMEN}

Objetivo: evaluar si existe una relación entre el número de tornillos pediculares (densidad de tornillos) y el porcentual de corrección de la curva principal en el tratamiento quirúrgico de la escoliosis neuromuscular. Métodos: fueron evaluados, retrospectivamente, $55 \mathrm{pa}$ cientes portadores de la escoliosis neuromuscular sometidos al tratamiento quirúrgico por medio de la artrodesis, exclusivamente por la vía posterior. Fueron analizados el valor de la curva preoperatoria, el porcentual de corrección y el valor de la curva postoperatoria en las radiografias pre y postoperatoria inmediata. Se calculó la densidad de tornillos (número de tornillos por pedículo en el área correspondiente a la curva principal) y se evaluó su relación con el porcentaje de corrección, por medio del análisis de corrección de Spearman. Resultados:

\footnotetext{
Trabalho realizado pelo Grupo de Escoliose da Associação de Assistência à Criança Deficiente - AACD - São Paulo (SP), Brasil.

Estagiário do Grupo de Escoliose da Associação de Assistência à Criança Deficiente - AACD - São Paulo (SP), Brasil.

2 Assistentes do Grupo de Escoliose da Associação de Assistência à Criança Deficiente - AACD - São Paulo (SP), Brasil.

${ }^{3}$ Chefe do Grupo de Escoliose da Associação de Assistência à Criança Deficiente - AACD - São Paulo (SP), Brasil. 
culino, com média de idade de 16,04 anos $(\mathrm{dp}=4,45)$. A doença de base mais frequente foi a paralisia cerebral. $\mathrm{O}$ valor da escoliose pré-operatória foi, em média, de $81,96^{\circ}(\mathrm{dp}=25,49)$ e da escoliose residual de $33,82^{\circ}$ $(\mathrm{dp}=19,02)$, com percentual de correção de $60,28 \%(d p=15,89)$. Houve uma relação positiva $(\mathrm{r}=0,266)$ e estatisticamente significante entre a densidade de parafusos e a correção da deformidade $(\mathrm{p}=0,045)$. Conclusões: no tratamento cirúrgico das deformidades neuromusculares existe uma relação positiva entre o maior número de parafusos dentro da área da curva principal e o percentual de correção.
27 (48\%) males were analyzed, with mean age of 16.04 years $(s d=4.45)$. The commonest base pathology was the cerebral palsy. The preoperative Cobb angle was $81.96^{\circ}(s d=25.49)$ and postoperative Cobb angle was $33.82^{\circ}, \quad(s d=19.02)$, with averaged curve correction of $60.28 \%$ $(s d=15.89)$. There was a significant correlation between screw density and curve correction ( $r=0.266$; $p=0.045)$. Conclusions: major curve correction correlates positively with the number of pedicular screw within the curve in the surgical treatment of neuromuscular scoliosis de los 55 pacientes, 28 (51\%) eran del sexo femenino y 27 (49\%) del masculino, con promedio de edad de 16.04 años ( $d p=4.45)$. La patología de base más común fue la parálisis cerebral. El valor de la escoliosis preoperatoria fue en promedio de $81.96^{\circ}(\mathrm{dp}=25.49)$ y de la escoliosis residual de $33.82^{\circ}(d p=19.02)$, con porcentual de corrección de $60.28 \%$ $(d p=15.89)$. Hubo una relación positiva $(r=0.266)$ y estadísticamente significativa entre la densidad de los tornillos y la corrección de la deformidad ( $p=0.045)$. Conclusiones: en el tratamiento quirúrgico de las deformidades neuromusculares, hay una relación positiva entre el mayor número de tornillos dentro del área de la curva principal y el porcentual de corrección.
DESCRITORES: Coluna vertebral/ patologia; Escoliose/cirurgia; Fusão vertebral/métodos; Parafusos ósseos; Doenças neuromusculares/cirurgia; Resultado de tratamento
KEYWORDS: Spine/pathology; Scoliosis/surgery; Spinal fusion/methods; Bone screws; Neuromuscular diseases/ surgery; Treatment outcome

\author{
DESCRIPTORES: Columna \\ vertebral/patología; \\ Escoliosis/cirugía; Fusión \\ vertebral/métodos; Tornillos \\ óseos; Enfermedades \\ neuromusculares/cirugía; \\ Resultado del tratamiento
}

\section{INTRODUÇÃO}

O início da instrumentação vertebral ocorreu em 1955, com as hastes de distração de Harrington ${ }^{1}$. Apesar de ser considerado um grande avanço na história da cirurgia da coluna, esse instrumental apresentava sérias limitações, principalmente relacionadas à necessidade de imobilização pós-operatória e à criação de deformidades no plano sagital ${ }^{2}$.

No final dos anos 1970, Luque apresentou outra inovação importante com seu sistema de fixação segmentar por meio de fios sublaminares. Com isso, ganhou-se muita estabilidade, diminuindo a necessidade de imobilização pós-operatória ${ }^{1,2}$.

Posteriormente, em 1984, Cotrel e Dubousset desenvolveram seu sistema de fixação por meio de ganchos que permitiam boa correção da deformidade, inclusive no plano axial, além de boa estabilidade pela fixação segmentar ${ }^{1}$.

Esse sistema permaneceu como padrão durante alguns anos até o surgimento dos parafusos pediculares que, primeiramente, foram utilizados na coluna lombar e, após a comprovação de sua segurança, têm sido cada vez mais utilizados na coluna torácica para correção de deformidades $^{3,4}$. Acredita-se que esses implantes possuam maior poder de correção e menor perda desta ao longo do tempo, além de permitirem a economia de níveis por meio de artrodeses mais curtas $^{4-6}$.

Apesar de utilizados inicialmente apenas no tratamento das escolioses idiopáticas, hoje há uma tendência em utilizá-los em curvas de alto valor angular, inclusive nas escolioses neuromusculares ${ }^{7,8}$.

Recentemente, Clements et al. ${ }^{9}$ apresentaram um trabalho que demonstrou a relação positiva entre o número de pontos de fixação (densidade de implante) e o percentual de correção obtida no tratamento da escoliose idiopática. Apesar de considerar vários tipos de fixação para a realização do estudo (fios, ganchos e parafusos), o grupo operado apenas com parafusos também apresentou maior percentual de correção.

O objetivo deste estudo foi avaliar a relação entre o número de implantes e o percentual de correção das escolioses neuromusculares submetidas ao tratamento cirúrgico com parafuso pedicular.

\section{MÉTODOS}

Foram avaliados retrospectivamente os prontuários e radiografias de 55 pacientes portadores de escoliose neuromuscular submetidos ao tratamento cirúrgico de maneira 
não consecutiva na Associação de Assistência à Criança Deficiente (AACD), em São Paulo (SP), no ano de 2008. Para manter a amostra a mais homogênea possível, foram excluídas as deformidades idiopáticas e congênitas.

Foram incluídos os casos submetidos à artrodese exclusivamente por via posterior, com a utilização apenas de parafusos pediculares. Em 20 (36\%) casos, foram utilizados um ou dois ganchos no nível de instrumentação mais cranial, na tentativa de aumentar a força de arrancamento desses implantes - sem interferir no estudo, já que estavam fora da área da curva principal.

A avaliação radiográfica foi realizada por meio de radiografias panorâmicas pré-operatórias da coluna vertebral, tiradas com o paciente em ortostatismo ou sentado com o mínimo apoio possível nos pacientes não deambuladores, e por radiografias panorâmicas no pós-operatório imediato. Foram consideradas apenas as radiografias no plano ântero-posterior, pois só foi avaliada a deformidade no plano coronal.

Os parâmetros analisados foram: valor angular préoperatório da curva principal, medido pelo método de Cobb (Cobb pré), Cobb pós-operatório (Cobb pós) e percentual de correção da deformidade (Correção $=$ Cobb pré - Cobb pós / Cobb pré x 100).

Também foi calculada o percentual de parafusos utilizados em relação ao número de possíveis pontos para a fixação de cada vértebra que fazia parte da curva, ou seja, dois por nível de Cobb a Cobb, que foi chamada de densidade de parafuso. Por exemplo, em uma curva de T12-L4 (cinco vértebras, dez pedículos), na qual se utilizam seis parafusos, a densidade de parafusos é de $60 \%$.

A correlação entre correção da curva e a densidade de parafusos foi realizada pela análise de correlação de Spearman, utilizando-se o programa Statistical Package for Social Sciences, versão 13.0. Foi considerado o nível de significância de $5 \%$.

\section{RESULTADOS}

Dos 55 pacientes, 27 (49\%) eram do sexo masculino, com média de idade de 16,04 anos $(\mathrm{dp}=4,45)$. Desses, a maioria tinha como diagnóstico principal a paralisia cerebral (PC), seguida de lesão medular (LM), mielomeningocele (MM) e amiotrofia espinhal (AME) (Gráfico 1).

Quanto ao tipo de curva, a mais comum foi a toracolombar, com 26 casos (47\%), seguida pelas curvas lombares (18 casos, 33\%), curvas torácicas ( 9 casos, $16 \%)$ e duplas curvas ( 2 casos, $4 \%$ ). Nessas, foi considerada apenas a curva principal para avaliação.

A média do valor angular pré-operatório foi de $81,96^{\circ}$ $(d p=25,49)$ com correção média de 60,28\% $(d p=15,89)$.

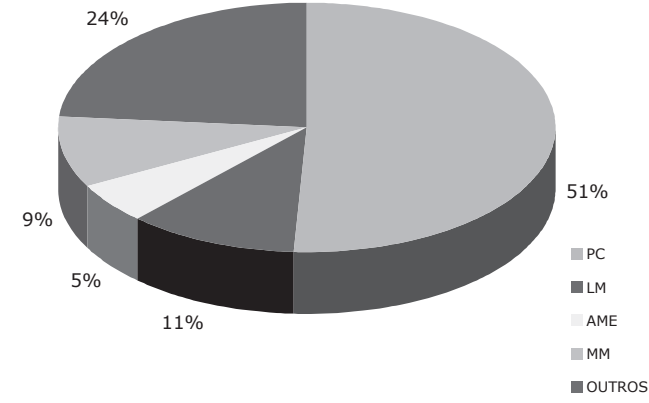

PC: paralisia cerebral; LM: lesão medular; AME: amiotrofia espinhal; MM: mielomeningocele.

Gráfico 1

Casuística dos pacientes de acordo com a patologia de base.

A média da curva residual foi de $33,82^{\circ}(\mathrm{dp}=19,02)$ (Tabela 1).

Por se tratar de pacientes neuromusculares, na maioria não deambuladores (46 casos, $84 \%$ ) e com obliquidade pélvica, o principal tipo de tratamento foi a artrodese longa, estendendo até o sacro, com fixação por meio de parafusos ilíacos (47 casos, $85 \%$ ).

A densidade de parafuso média foi de 59,91\% $(\mathrm{dp}=12,22)$, com valores mínimo e máximo de 33,33 e $91,66 \%$, respectivamente.

Quando relacionado ao percentual de correção da escoliose e a densidade de parafusos, foi constatado uma correlação positiva de 0,266 , estatisticamente significante $(\mathrm{p}=0,049)$, conforme o Gráfico 2 .

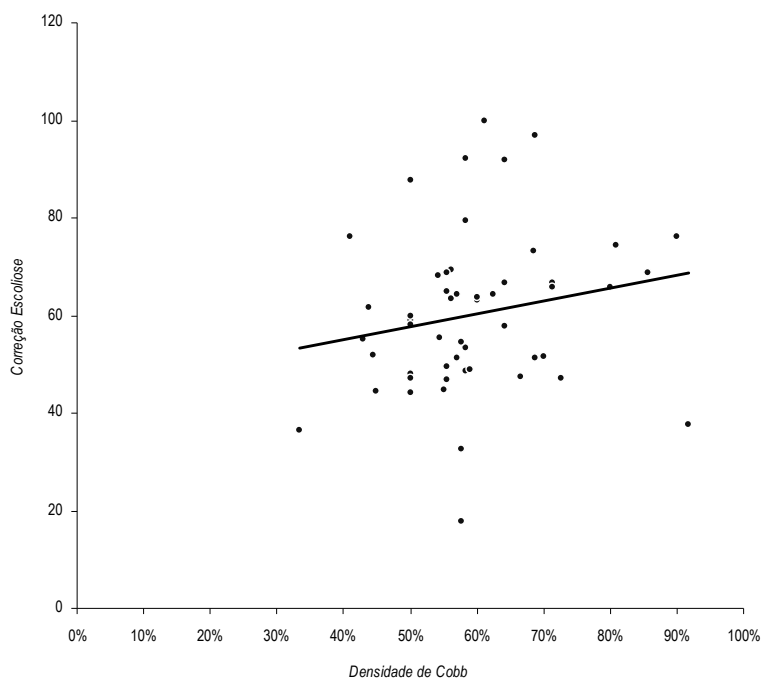

Gráfico 2

Correlação entre o percentual de correção da escoliose e a densidade de parafusos.

\section{TABELA 1 - Valores pré e pós-operatório e percentual de correção das curvas submetidos a tratamento cirúrgico}

\begin{tabular}{lcccc}
\hline Variável & Mínimo & Máximo & Média & Desvio padrão \\
\hline Escoliose pré-operatório (Cobb pré) & $33,00^{\circ}$ & $143,00^{\circ}$ & $81,96^{\circ}$ & 25,49 \\
Correção & $17,64 \%$ & $100 \%$ & $60,28 \%$ & 15,89 \\
Escoliose pós-operatório (Cobb pós) & $0,00^{\circ}$ & $84,00^{\circ}$ & $33,82^{\circ}$ & 19,02 \\
\hline
\end{tabular}




\section{DISCUSSÃO}

Recentemente, vários avanços foram realizados no campo da cirurgia da coluna vertebral, principalmente em relação aos tipos de implantes ${ }^{1}$. Com o advento do parafuso pedicular e a sua utilização na correção de deformidades, vários estudos demonstram suas vantagens em relação aos demais sistemas de fixação, entre elas o maior poder de correção, a menor perda desta ao longo do tempo e o menor índice de pseudoartrose $5,6,10$.

Este trabalho mostrou um percentual de correção médio de $60,28 \%$ - um pouco abaixo da média encontrada na literatura ${ }^{2}$, porém semelhante aos encontrados no tratamento de deformidades neuromusculares utilizando parafusos ${ }^{7,8}$. Isso talvez se explique pelo maior valor angular e pela rigidez das deformidades encontradas nesses pacientes, já que a média da magnitude das curvas em nosso estudo foi de $81,96^{\circ}$.

Assim como Clements et al. ${ }^{9}$ encontraram uma relação positiva entre a correção no plano coronal e o número de implantes utilizados na área da curva principal, independente do tipo de material utilizado, no presente estudo, também foi encontrada essa relação entre a densidade do parafuso e o percentual de correção da escoliose. Isso mostra que o maior número de vértebras instrumentadas no ápice da deformidade pode aumentar o poder de correção do material, o que pode ser bastante útil, principalmente no tratamento de deformidades graves, já que, com esta estratégia, pode-se diminuir a necessidade de uma abordagem por via anterior para aumentar a flexibilidade da curva, o que agrega bastante morbidade ao procedimento. Esse fato tem ainda mais relevância quando se leva em consideração os pacientes com doenças neuromusculares, que, pelas características associadas, já apresentam uma capacidade pulmonar reduzida.

Em 2005, Luhman et al. ${ }^{11}$ compararam os pacientes com escoliose idiopática do adolescente com curvas entre 70 e $100^{\circ}$ submetidos à artrodese por via posterior e aqueles nos quais foi realizada uma liberação por via anterior, além do procedimento posterior. No grupo operado apenas pela via posterior, foram utilizados ganchos, montagens híbridas e construções com parafusos apenas. Os resultados mostraram um maior percentual de correção no grupo submetido à dupla abordagem, porém, quando os casos apenas com parafusos foram considerados isoladamente em relação ao grupo da dupla via, não houve diferença significativa. Isso mostra a tendência cada vez menor de realização de dupla abordagem no tratamento de deformidades de alto valor angular com a utilização do parafuso pedicular.

O principal objetivo do tratamento cirúrgico das deformidades vertebrais é manter um tronco compensado ${ }^{12}$, principalmente quando se leva em consideração pacientes com doenças neuromusculares. No entanto, o maior poder de correção dos parafusos pediculares pode agregar melhoras no cuidado desses pacientes, como, por exemplo, a diminuição das cirurgias pela via anterior, que tanto aumentam a morbidade do tratamento.

\section{CONCLUSÕES}

No tratamento cirúrgico das escolioses neuromusculares, o percentual de correção da curva principal se relaciona positivamente à densidade de parafusos na área da curva, ou seja, a correção da curva aumenta com a maior quantidade de implantes por vértebra na área da curva.

\section{REFERÊNCIAS}

1. Moen KY, Nachemson AL. Treatment of scoliosis. An historical perspective. Spine. 1999;24(24):2570-5.

2. Lenke LG, Kuklo TR, Ondra S, Polly DW Jr. Rationale behind the current state-of-the-art treatment of scoliosis (in the pedicle screw era). Spine. 2008;33(10):1051-4. Comment on: Spine. 2007;32(24):2641-3.

3. Kim YJ, Lenke LG, Bridwell KH, Cho YS, Riew KD. Free hand pedicle screw placement in the thoracic spine: is it safe? Spine. 2004;29(3):333-42; discussion 342.

4. Suk SI, Lee Ck, Kim WJ, Chung YJ, Park YB. Segmental pedicle screw fixation in the treatment of thoracic idiopathic scoliosis. Spine. 1995;20(12):1399-405.
5. Kim YJ, Lenke LG, Kim J, Bridwell $\mathrm{KH}$, Cho SK, Cheh G, et al. Comparative analysis of pedicle screw versus hybrid instrumentation in posterior fusion of adolescent idiopathic scoliosis. Spine. 2006;31(3):291-8.

6. Liljenqvist U, Lepsien U, Hackenberg L, Niemeyer T, Halm H. Comparative analysis of pedicle screw and hook instrumentation in posterior correction and fusion of idiopathic thoracic scoliosis. Eur Spine J. 2002;11(4):336-43.

7. Oliveira GC, Cavali PTM, Landim E, Santos MAM, Lehoczki MA. Instrumental de $3^{\text {a }}$ geração no tratamento de escoliose em pacientes com paralisia cerebral tetraparéticos - análise dos resultados clínico e radiográfico. Coluna/Columna. 2007;6(4):201-10.
8. Modi HN, Suh SW, Song HR, Fernandez HM, Yang JH. Treatment of neuromuscular scoliosis with posterior-only pedicle screw fixation. J Orthop Surg Res. 2008;3:23-31.

9. Clements D, Betz RR, Newton PO, Marks MC, Bastrom T, Porter S. Correlation of scoliosis curve correction with the number and type of fixation anchors. In: 42 ${ }^{\text {sd }}$ SRS Annual Meeting; 2007 Sept 5-8; Edinburgh, Scotland.

10.Mulpuri K, Perdios A, Reilly CW. Evidence-based medicine analysis of all pedicle screw constructs in adolescent idiopathic scoliosis. Spine. 2007;32(19 Suppl):S109-14. 
11.Luhmann SJ, Lenke LG, Kim YJ, Bridwell KH, Schootman M. Thoracic adolescent idiopathic scoliosis curves between 70 degrees and 100 degrees: is anterior release necessary? Spine. 2005;30(18):2061-7.
12. Winter RB, Lonstein JE, Denis F. How much correction is enough? Spine. 2007;32(24):2641-3. Comment in: Spine. 2008;33(10):1051-4.

\section{Correspondência}

Murilo Tavares Daher

Rua Martinico Prado, 71, apto. 24 Vila Buarque

CEP: 01224-010 - São Paulo (SP), Brasil

Tel: (1 1) 7542-6270/62 3281-2397

E-mail:murilodaher@uol.com.br 